Acta Crystallographica Section E

Structure Reports

Online

ISSN 1600-5368

\section{2,6-Bis(4H-1,2,4-triazol-4-yl)pyridine dihydrate}

\section{De-quan Jiao, Xiao Tong Han, Qiong Zhou and Ying Wang*}

Tianjin Key Laboratory of Structure and Performances of Functional Molecules Tianjin Normal University, Tianjin 300387, People's Republic of China

Correspondence e-mail: wangying790601@163.com

Received 8 September 2011; accepted 10 October 2011

Key indicators: single-crystal X-ray study; $T=566 \mathrm{~K}$; mean $\sigma(\mathrm{C}-\mathrm{C})=0.004 \AA$; $R$ factor $=0.051 ; w R$ factor $=0.133 ;$ data-to-parameter ratio $=11.5$.

The asymmetric unit of the title compound, $\mathrm{C}_{9} \mathrm{H}_{7} \mathrm{~N}_{7} \cdot 2 \mathrm{H}_{2} \mathrm{O}$, comprises three formula units. The dihedral angles between the triazole rings and the respective central pyridine rings are 4.87 (16)/1.39 (17), 6.46 (16)/7.61 (16) and $7.00(16) / 3.77(17)^{\circ}$. The water molecules form $\mathrm{O}-\mathrm{H} \cdots \mathrm{O}$ hydrogen bonds between themselves and $\mathrm{O}-\mathrm{H} \cdots \mathrm{N}$ hydrogen bonds with the $\mathrm{N}$-atom acceptors of the triazole rings, producing a threedimensional framework.

\section{Related literature}

For the synthesis of the title compound, see: Wiley \& Hart (1953). For properties of related compounds, see: Haasnoot (2000).

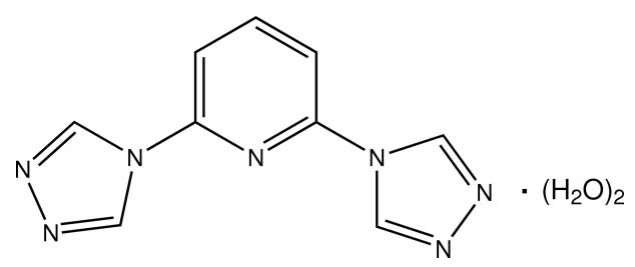

\section{Experimental}

Crystal data

$\mathrm{C}_{9} \mathrm{H}_{7} \mathrm{~N}_{7} \cdot 2 \mathrm{H}_{2} \mathrm{O}$

$M_{r}=249.25$

Monoclinic, $P 2_{1} / c$

$a=9.7211(17) \AA$

$b=17.921(3) \AA$

$c=19.603(4) \AA$

$\beta=91.333$ (3)
Data collection

Bruker SMART CCD area-detector diffractometer

Absorption correction: multi-scan (SADABS; Sheldrick, 1996)

$T_{\min }=0.966, T_{\max }=0.991$

Refinement

$R\left[F^{2}>2 \sigma\left(F^{2}\right)\right]=0.051$

$w R\left(F^{2}\right)=0.133$

$S=1.00$

6030 reflections

523 parameters

18 restraints

19614 measured reflections 6030 independent reflections 3151 reflections with $I>2 \sigma(I)$ $R_{\text {int }}=0.057$

$\mathrm{H}$ atoms treated by a mixture of independent and constrained refinement

$\Delta \rho_{\max }=0.17{\mathrm{e} \AA^{-3}}^{-3}$

$\Delta \rho_{\min }=-0.25{\mathrm{e} \AA^{-3}}^{-3}$

Table 1

Hydrogen-bond geometry $\left(\AA{ }^{\circ}\right)$.

\begin{tabular}{|c|c|c|c|c|}
\hline$D-\mathrm{H} \cdots A$ & $D-\mathrm{H}$ & $\mathrm{H} \cdots A$ & $D \cdots A$ & $D-\mathrm{H} \cdots A$ \\
\hline $\mathrm{O} 6-\mathrm{H} 6 A \cdots \mathrm{N} 21^{\mathrm{i}}$ & $0.86(1)$ & $1.95(1)$ & $2.801(3)$ & $172(3)$ \\
\hline $\mathrm{O} 6-\mathrm{H} 6 B \cdots \mathrm{N} 1^{\mathrm{ii}}$ & $0.86(1)$ & $2.04(1)$ & $2.878(3)$ & $166(3)$ \\
\hline $\mathrm{O} 5-\mathrm{H} 5 A \cdots \mathrm{O} 6$ & $0.87(1)$ & $1.86(2)$ & 2.707 (3) & $166(4)$ \\
\hline $\mathrm{O} 5-\mathrm{H} 5 B \cdots \mathrm{O} 1^{\mathrm{iii}}$ & $0.86(1)$ & $2.01(1)$ & $2.864(4)$ & $171(4)$ \\
\hline $\mathrm{O} 4-\mathrm{H} 4 A \cdots \mathrm{N} 14^{\mathrm{iv}}$ & $0.86(1)$ & $1.95(1)$ & $2.808(3)$ & $173(4)$ \\
\hline $\mathrm{O} 4-\mathrm{H} 4 B \cdots \mathrm{N} 8^{\mathrm{v}}$ & $0.86(1)$ & $2.09(2)$ & $2.907(3)$ & $160(3)$ \\
\hline $\mathrm{O} 3-\mathrm{H} 3 B \cdots \mathrm{O} 5$ & $0.86(1)$ & $2.03(2)$ & $2.868(4)$ & $166(4)$ \\
\hline $\mathrm{O} 3-\mathrm{H} 3 A \cdots \mathrm{O} 4$ & $0.85(1)$ & $2.01(1)$ & $2.855(3)$ & $173(3)$ \\
\hline $\mathrm{O} 2-\mathrm{H} 2 B \cdots \mathrm{O} 4$ & $0.86(1)$ & $2.00(1)$ & $2.851(4)$ & $172(4)$ \\
\hline $\mathrm{O} 2-\mathrm{H} 2 A \cdots \mathrm{O} 1$ & $0.87(1)$ & $2.03(1)$ & $2.886(3)$ & $171(3)$ \\
\hline $\mathrm{O} 1-\mathrm{H} 1 B \cdots \mathrm{N} 15^{\mathrm{vi}}$ & $0.86(1)$ & $2.05(2)$ & $2.861(3)$ & $158(3)$ \\
\hline $\mathrm{O} 1-\mathrm{H} 1 A \cdots \mathrm{N} 7$ & $0.86(1)$ & $1.97(1)$ & $2.820(3)$ & $171(3)$ \\
\hline
\end{tabular}

Symmetry codes: (i) $x-1, y, z$; (ii) $-x, y+\frac{1}{2},-z+\frac{3}{2}$; (iii) $x,-y+\frac{3}{2}, z-\frac{1}{2}$; (iv) $-x+2, y-\frac{1}{2},-z+\frac{3}{2} ;(\mathrm{v}) x+1, y, z ;(\mathrm{vi})-x+1, y+\frac{1}{2},-z+\frac{3}{2}$.

Data collection: SMART (Bruker, 2008); cell refinement: SAINT (Bruker, 2008); data reduction: $S A I N T$; program(s) used to solve structure: SHELXS97 (Sheldrick, 2008); program(s) used to refine structure: SHELXL97 (Sheldrick, 2008); molecular graphics: SHELXTL (Sheldrick, 2008); software used to prepare material for publication: SHELXTL.

This work was supported financially by Tianjin Normal University (grant No. 5RL090), the Natural Science Foundation of Tianjin (grant No. 11JCYBJC03600) and the Young Scientist Fund (grant No. 52 G10005).

Supplementary data and figures for this paper are available from the IUCr electronic archives (Reference: GO2026).

\title{
References
}

Bruker (2008). APEX2 and SAINT. Bruker AXS Inc., Madison, Wisconsin, USA.

Haasnoot, J. G. (2000). Coord. Chem. Rev. 200, 131-185.

Sheldrick, G. M. (1996). SADABS. University of Göttingen, Germany.

Sheldrick, G. M. (2008). Acta Cryst. A64, 112-122.

Wiley, R. H. \& Hart, A. J. (1953). J. Org. Chem. 18, 1368-1371. 


\section{supporting information}

Acta Cryst. (2011). E67, o2944 [doi:10.1107/S1600536811041687]

\section{2,6-Bis(4H-1,2,4-triazol-4-yl)pyridine dihydrate}

\section{De-quan Jiao, Xiao Tong Han, Qiong Zhou and Ying Wang}

\section{S1. Comment}

Many molecular-based compounds exhibit interesting magnetic and luminescent properties, (Haasnoot, 2000). One of the requirements for producing such macroscopic properties is to create interactions between the molecular units and the active sites within the crystal lattices. 1,2,4-triazole and, in particular, its derivatives are interesting bridging ligands.

The asymmetric unit of (I) comprises three $\mathrm{C}_{9} \mathrm{H}_{7} \mathrm{~N}_{7} .2 \mathrm{H}_{2} \mathrm{O}$ components of those ilustrated in Figure 1. The dihedral angles between the triazole rings and the respective central pyridine rings are (atoms named indicate the relevant rings) $\mathrm{N} 3 / \mathrm{N} 44.87(16)^{\circ}, \mathrm{N} 4 / \mathrm{N} 51.39(17)^{\circ}, \mathrm{N} 10 / \mathrm{N} 116.46(16)^{\circ}, \mathrm{N} 11 / \mathrm{N} 127.61(16)^{\circ}, \mathrm{N} 17 / \mathrm{N} 187.00(16)^{\circ}$ and N18/N19 $3.77(17)^{\circ}$. The water molecules form $\mathrm{O}-\mathrm{H} \cdots \mathrm{O}$ hydrogen bonds between themselves and $\mathrm{O}-\mathrm{H}^{\cdots} \cdots \mathrm{N}$ bonds with the $\mathrm{N}$ atom acceptors of the triazole rings, producing a three-dimensional framework. (Table 1, Figure 2).

\section{S2. Experimental}

A mixture of $1.3 \mathrm{~g}(0.012 \mathrm{~mol})$ of 2,6-diaminopyridine and $2.0 \mathrm{~g}(0.023 \mathrm{~mol})$ of diformylhydrazine was heated slowly to $160-170{ }^{\circ} \mathrm{C}$ for $30 \mathrm{~min}$. The crystals, which separated on cooling, were collected and recrystallized form water and acetonitrile to give $0.8 \mathrm{~g}$ of (I) (yield 20\%). After several recrystallizations from water, the air-dried product was obtained as white needles, m.p. 325-327 K (placed in hot bolck at $320 \mathrm{~K}$ ). The analysis was obtained on the air-dried sample. Anal. Calc. for $\mathrm{C}_{9} \mathrm{H}_{11} \mathrm{~N}_{7} \mathrm{O}_{2}$ (\%): C, 46.75; H, 3.92. Found (\%): C, 46.89; H, 4.00.

\section{S3. Refinement}

Positional parameters of all the $\mathrm{H}$ atoms were calculated geometrically and were allowed to ride on the $\mathrm{C}$ or $\mathrm{O}$ atoms with $\mathrm{C}-\mathrm{H}=0.93 \AA$ and $\mathrm{O}-\mathrm{H}=0.85 \AA$ and $U$ iso $(\mathrm{H})=1.2$ or 1.5 times $U$ eq $(\mathrm{C}$ or $\mathrm{O})$. The hydrogen atoms of the water molecules were located from difference maps and refined with isotropic temperature factors.In the case of atoms $\mathrm{O} 2$ and $\mathrm{O} 3$ the angle between the $\mathrm{H}$ atoms was restained to be a value close to $104^{\circ}$. 


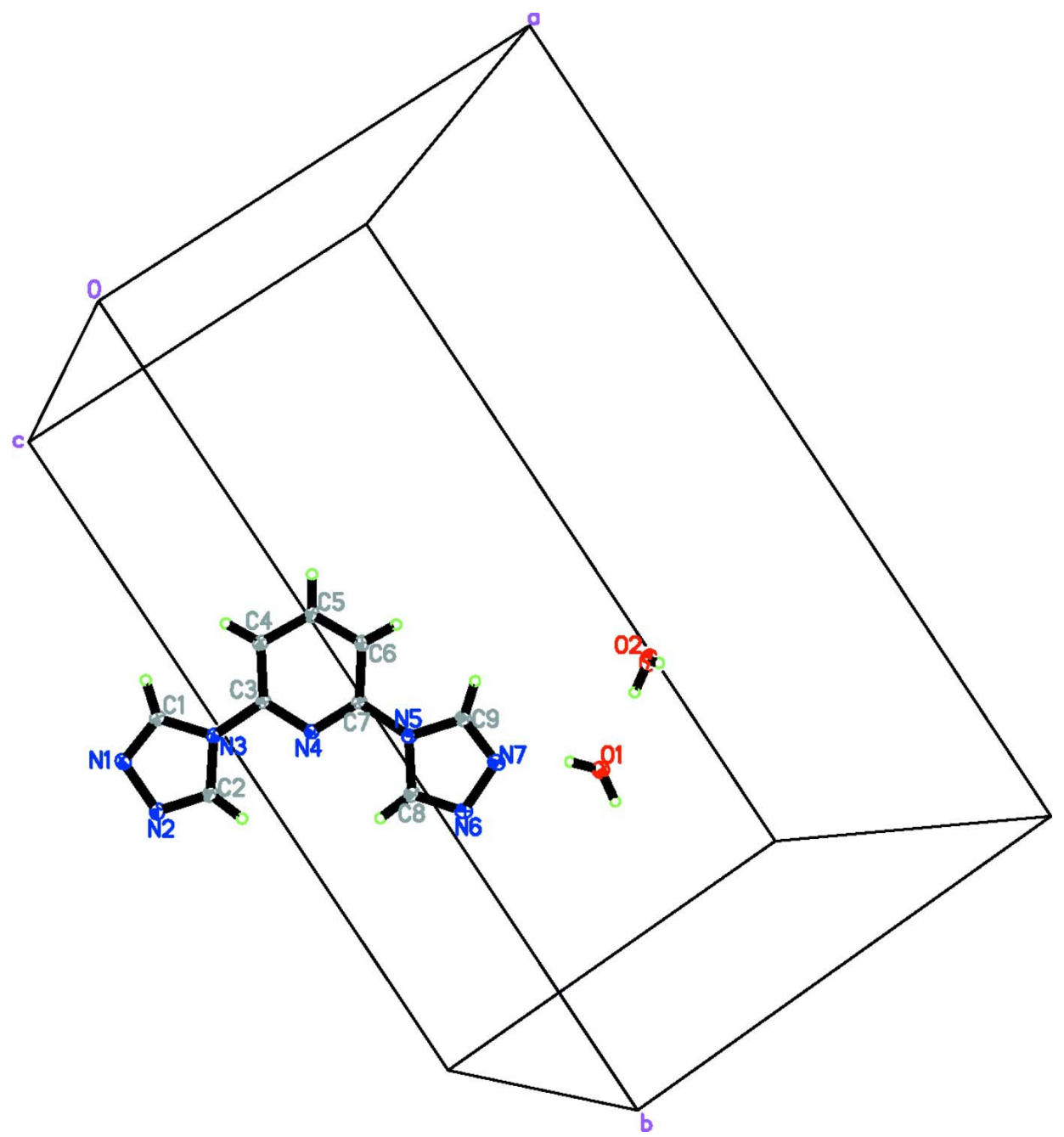

Figure 1

View of the molecule of (I) showing the atom-labelling scheme. Displacement ellipsoids are drawn at the $50 \%$ probability level. 


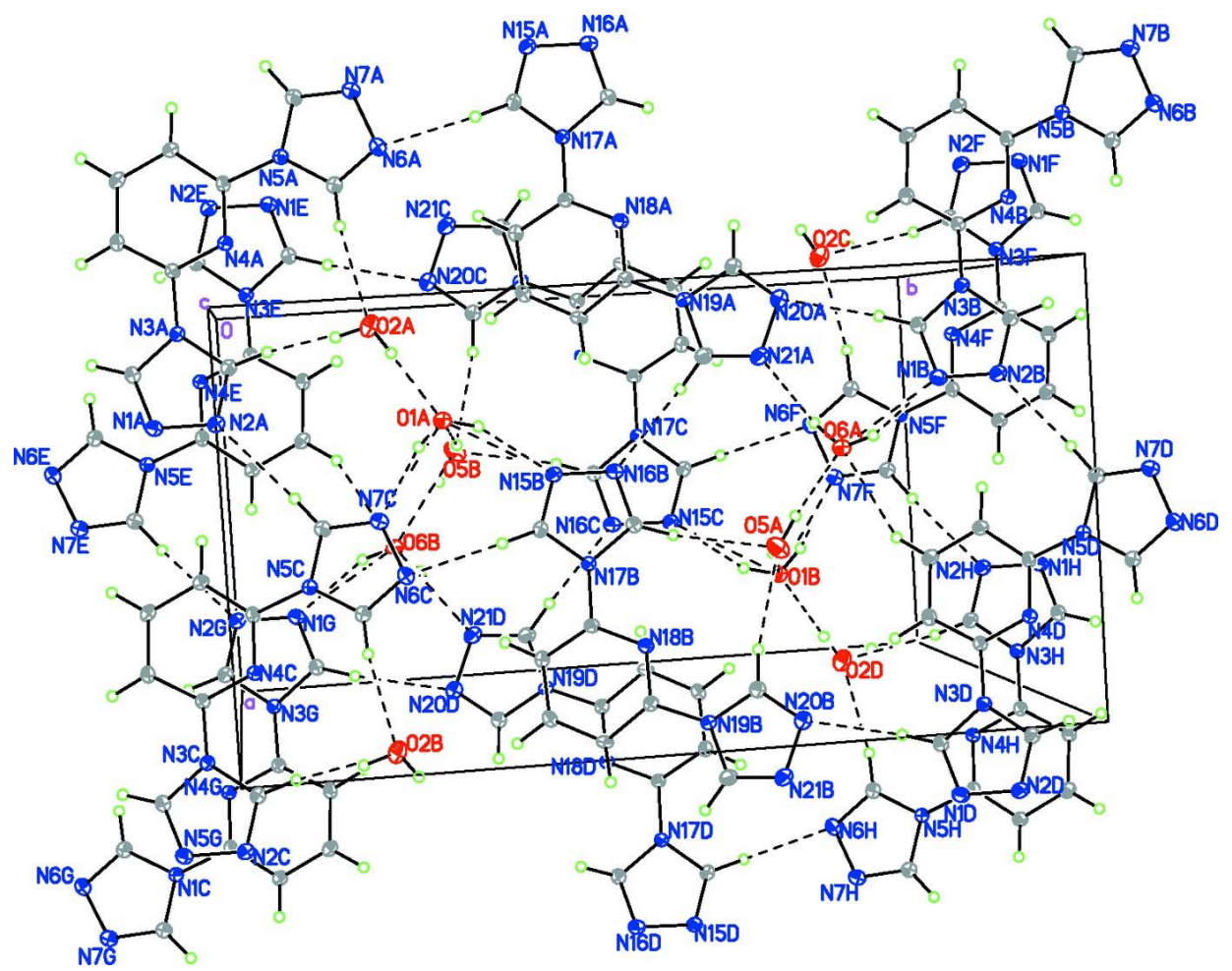

\section{Figure 2}

The crystal packing for (I), with the $\mathrm{H}$ bond interactions shown as dashed lines.

\section{2,6-Bis(4H-1,2,4-triazol-4-yl)pyridine dihydrate}

Crystal data

$\mathrm{C}_{9} \mathrm{H}_{7} \mathrm{~N}_{7} \cdot 2 \mathrm{H}_{2} \mathrm{O}$

$M_{r}=249.25$

Monoclinic, $P 2{ }_{1} / c$

$a=9.7211(17) \AA$

$b=17.921(3) \AA$

$c=19.603(4) \AA$

$\beta=91.333(3)^{\circ}$

$V=3414.2(10) \AA^{3}$

$Z=12$

\section{Data collection}

Bruker SMART CCD area-detector diffractometer

Radiation source: fine-focus sealed tube Graphite monochromator $\varphi$ and $\omega$ scans

Absorption correction: multi-scan

(SADABS; Sheldrick, 1996)

$T_{\min }=0.966, T_{\max }=0.991$
$F(000)=1560$

$D_{\mathrm{x}}=1.455 \mathrm{Mg} \mathrm{m}^{-3}$

Mo $K \alpha$ radiation, $\lambda=0.71073 \AA$

Cell parameters from 1505 reflections

$\theta=2.4-21.4^{\circ}$

$\mu=0.11 \mathrm{~mm}^{-1}$

$T=566 \mathrm{~K}$

PLATE, colorless

$0.32 \times 0.16 \times 0.08 \mathrm{~mm}$

19614 measured reflections

6030 independent reflections

3151 reflections with $I>2 \sigma(I)$

$R_{\text {int }}=0.057$

$\theta_{\max }=25.0^{\circ}, \theta_{\min }=2.1^{\circ}$

$h=-11 \rightarrow 11$

$k=-21 \rightarrow 21$

$l=-23 \rightarrow 18$ 


\section{Refinement}

Refinement on $F^{2}$

Least-squares matrix: full

$R\left[F^{2}>2 \sigma\left(F^{2}\right)\right]=0.051$

$w R\left(F^{2}\right)=0.133$

$S=1.00$

6030 reflections

523 parameters

18 restraints

Primary atom site location: structure-invariant direct methods
Secondary atom site location: difference Fourier map

Hydrogen site location: inferred from neighbouring sites

$\mathrm{H}$ atoms treated by a mixture of independent and constrained refinement

$w=1 /\left[\sigma^{2}\left(F_{\mathrm{o}}^{2}\right)+(0.0378 P)^{2}+0.9562 P\right]$ where $P=\left(F_{\mathrm{o}}{ }^{2}+2 F_{\mathrm{c}}{ }^{2}\right) / 3$

$(\Delta / \sigma)_{\max }<0.001$

$\Delta \rho_{\max }=0.17 \mathrm{e} \AA^{-3}$

$\Delta \rho_{\min }=-0.25 \mathrm{e} \AA^{-3}$

\section{Special details}

Geometry. All e.s.d.'s (except the e.s.d. in the dihedral angle between two 1.s. planes) are estimated using the full covariance matrix. The cell e.s.d.'s are taken into account individually in the estimation of e.s.d.'s in distances, angles and torsion angles; correlations between e.s.d.'s in cell parameters are only used when they are defined by crystal symmetry. An approximate (isotropic) treatment of cell e.s.d.'s is used for estimating e.s.d.'s involving 1.s. planes.

Refinement. Refinement of $F^{2}$ against ALL reflections. The weighted $R$-factor $w R$ and goodness of fit $S$ are based on $F^{2}$, conventional $R$-factors $R$ are based on $F$, with $F$ set to zero for negative $F^{2}$. The threshold expression of $F^{2}>\sigma\left(F^{2}\right)$ is used only for calculating $R$-factors(gt) $e t c$. and is not relevant to the choice of reflections for refinement. $R$-factors based on $F^{2}$ are statistically about twice as large as those based on $F$, and $R$ - factors based on ALL data will be even larger.

Fractional atomic coordinates and isotropic or equivalent isotropic displacement parameters $\left(\AA^{2}\right)$

\begin{tabular}{|c|c|c|c|c|}
\hline & $x$ & $y$ & $z$ & $U_{\text {iso }} * / U_{\text {eq }}$ \\
\hline $\mathrm{O} 1$ & $0.7154(2)$ & $0.78220(11)$ & $0.96334(15)$ & $0.0661(7)$ \\
\hline H1A & $0.654(3)$ & $0.7512(12)$ & 0.9490 (19) & $0.099 *$ \\
\hline H1B & $0.694(3)$ & $0.8260(8)$ & $0.9486(19)$ & $0.099 *$ \\
\hline $\mathrm{O} 2$ & $0.9471(3)$ & $0.69444(15)$ & $0.92087(15)$ & $0.0823(8)$ \\
\hline $\mathrm{H} 2 \mathrm{~A}$ & $0.879(3)$ & $0.719(2)$ & $0.9382(16)$ & $0.123^{*}$ \\
\hline $\mathrm{H} 2 \mathrm{~B}$ & $0.943(4)$ & $0.701(2)$ & $0.8775(6)$ & $0.123 *$ \\
\hline $\mathrm{O} 3$ & 0.7061 & $0.61811(12)$ & 0.72477 (16) & $0.0778(8)$ \\
\hline $\mathrm{H} 3 \mathrm{~A}$ & $0.777(3)$ & $0.6395(18)$ & $0.7419(18)$ & $0.117^{*}$ \\
\hline H3B & $0.673(4)$ & $0.6445(18)$ & $0.6918(15)$ & $0.117^{*}$ \\
\hline $\mathrm{O} 4$ & $0.9378(2)$ & $0.70073(12)$ & $0.77552(15)$ & $0.0677(7)$ \\
\hline $\mathrm{H} 4 \mathrm{~A}$ & $1.004(2)$ & $0.6707(13)$ & 0.7666 (19) & $0.102 *$ \\
\hline H4B & $0.960(3)$ & $0.7445(8)$ & $0.762(2)$ & $0.102 *$ \\
\hline $\mathrm{O} 5$ & $0.6322(3)$ & $0.69516(15)$ & $0.60102(15)$ & $0.0821(8)$ \\
\hline H5A & $0.558(3)$ & $0.719(2)$ & $0.6101(18)$ & $0.123^{*}$ \\
\hline H5B & $0.648(4)$ & $0.701(2)$ & $0.5583(8)$ & $0.123^{*}$ \\
\hline O6 & $0.4062(2)$ & $0.78247(12)$ & $0.60995(15)$ & $0.0728(8)$ \\
\hline H6A & $0.341(3)$ & $0.7504(13)$ & $0.606(2)$ & $0.109 *$ \\
\hline H6B & $0.375(3)$ & $0.8260(8)$ & $0.600(2)$ & $0.109 *$ \\
\hline N1 & $-0.2572(2)$ & $0.41842(13)$ & $0.91734(13)$ & $0.0467(7)$ \\
\hline N2 & -0.2565 (2) & $0.49602(13)$ & $0.91358(13)$ & $0.0471(7)$ \\
\hline N3 & $-0.0433(2)$ & $0.45570(11)$ & $0.91796(12)$ & $0.0364(6)$ \\
\hline N4 & $0.1588(2)$ & $0.52262(11)$ & $0.91983(11)$ & $0.0339(6)$ \\
\hline N5 & $0.3497(2)$ & $0.59867(12)$ & $0.92021(12)$ & $0.0386(6)$ \\
\hline N6 & 0.3602 & $0.72068(13)$ & $0.92221(15)$ & $0.0541(8)$ \\
\hline
\end{tabular}




\begin{tabular}{|c|c|c|c|c|}
\hline N7 & 0.4924 (2) & $0.69199(13)$ & 0.91984 (15) & $0.0578(8)$ \\
\hline N8 & $0.0804(2)$ & $0.83897(12)$ & 0.74510 (14) & $0.0474(7)$ \\
\hline N9 & $0.0826(2)$ & $0.91653(12)$ & $0.74574(13)$ & $0.0448(7)$ \\
\hline N10 & $0.2947(2)$ & 0.87465 (11) & $0.75369(12)$ & $0.0352(6)$ \\
\hline N11 & 0.4984 (2) & 0.94024 (11) & 0.75457 (12) & $0.0337(6)$ \\
\hline N12 & $0.6900(2)$ & $1.01533(11)$ & $0.75413(12)$ & $0.0362(6)$ \\
\hline N13 & $0.7018(2)$ & $1.13674(13)$ & $0.74646(13)$ & $0.0490(7)$ \\
\hline N14 & 0.8339 (2) & $1.10798(13)$ & $0.74444(13)$ & $0.0477(7)$ \\
\hline N15 & $0.4265(2)$ & $0.41749(12)$ & $0.57083(13)$ & 0.0453 \\
\hline N16 & 0.4285 (2) & $0.49419(12)$ & $0.58098(13)$ & $0.0444(7)$ \\
\hline N17 & $0.6411(2)$ & $0.45391(11)$ & $0.57956(12)$ & $0.0339(6)$ \\
\hline N18 & $0.8443(2)$ & $0.52004(12)$ & $0.58430(12)$ & $0.0355(6)$ \\
\hline N19 & $1.0366(2)$ & $0.59472(12)$ & $0.58859(12)$ & $0.0391(6)$ \\
\hline N20 & $1.0484(3)$ & $0.71698(13)$ & $0.58745(16)$ & $0.0625(8)$ \\
\hline N21 & $1.1808(3)$ & $0.68779(14)$ & 0.58657 (14) & $0.0560(8)$ \\
\hline $\mathrm{C} 1$ & $-0.1310(3)$ & $0.39664(15)$ & $0.92001(16)$ & $0.0433(8)$ \\
\hline H1 & -0.1034 & 0.3470 & 0.9230 & $0.052 *$ \\
\hline $\mathrm{C} 2$ & $-0.1282(3)$ & $0.51609(15)$ & $0.91468(15)$ & $0.0419(8)$ \\
\hline $\mathrm{H} 2$ & -0.0981 & 0.5653 & 0.9134 & $0.050 *$ \\
\hline $\mathrm{C} 3$ & $0.1020(3)$ & 0.45514 (14) & 0.91807 (15) & $0.0352(7)$ \\
\hline $\mathrm{C} 4$ & $0.1745(3)$ & $0.38948(15)$ & $0.91611(16)$ & $0.0484(9)$ \\
\hline $\mathrm{H} 4$ & 0.1304 & 0.3434 & 0.9150 & $0.058 *$ \\
\hline $\mathrm{C} 5$ & $0.3165(3)$ & $0.39510(16)$ & 0.91587 (18) & $0.0543(9)$ \\
\hline H5 & 0.3698 & 0.3521 & 0.9145 & $0.065^{*}$ \\
\hline C6 & $0.3790(3)$ & $0.46336(14)$ & 0.91766 (16) & $0.0470(9)$ \\
\hline H6 & 0.4743 & 0.4679 & 0.9178 & $0.056^{*}$ \\
\hline $\mathrm{C} 7$ & 0.2948 (3) & $0.52521(14)$ & 0.91926 (14) & $0.0344(7)$ \\
\hline $\mathrm{C} 8$ & $0.2778(3)$ & $0.66368(15)$ & $0.92221(16)$ & $0.0462(8)$ \\
\hline H8 & 0.1824 & 0.6669 & 0.9234 & $0.055^{*}$ \\
\hline C9 & 0.4838 (3) & 0.61994 (16) & $0.91853(16)$ & $0.0486(9)$ \\
\hline H9 & 0.5583 & 0.5875 & 0.9167 & $0.058^{*}$ \\
\hline $\mathrm{C} 10$ & $0.2067(3)$ & $0.81618(15)$ & $0.74954(16)$ & $0.0457(8)$ \\
\hline H10 & 0.2334 & 0.7663 & 0.7498 & $0.055^{*}$ \\
\hline $\mathrm{C} 11$ & $0.2113(3)$ & $0.93586(15)$ & 0.75085 (14) & $0.0398(7)$ \\
\hline H11 & 0.2424 & 0.9849 & 0.7524 & $0.048^{*}$ \\
\hline C12 & $0.4406(2)$ & $0.87350(14)$ & $0.75887(14)$ & $0.0334(7)$ \\
\hline $\mathrm{C} 13$ & $0.5117(3)$ & $0.80762(15)$ & $0.76775(16)$ & $0.0452(8)$ \\
\hline H13 & 0.4666 & 0.7620 & 0.7705 & $0.054^{*}$ \\
\hline $\mathrm{C} 14$ & $0.6524(3)$ & $0.81262(15)$ & $0.77231(17)$ & $0.0488(9)$ \\
\hline H14 & 0.7046 & 0.7696 & 0.7787 & $0.059 *$ \\
\hline $\mathrm{C} 15$ & $0.7168(3)$ & $0.88044(15)$ & $0.76760(16)$ & 0.0430 \\
\hline H15 & 0.8122 & 0.8845 & 0.7698 & $0.052 *$ \\
\hline $\mathrm{C} 16$ & 0.6338 (3) & $0.94236(14)$ & $0.75953(14)$ & $0.0347(7)$ \\
\hline $\mathrm{C} 17$ & $0.6190(3)$ & $1.08051(15)$ & $0.75224(16)$ & $0.0437(8)$ \\
\hline H17 & 0.5238 & 1.0843 & 0.7548 & $0.052 *$ \\
\hline $\mathrm{C} 18$ & $0.8236(3)$ & $1.03616(15)$ & $0.74905(15)$ & $0.0426(8)$ \\
\hline H18 & 0.8976 & 1.0033 & 0.7489 & $0.051^{*}$ \\
\hline C19 & $0.5535(3)$ & $0.39572(15)$ & $0.57048(16)$ & $0.0436(8)$ \\
\hline
\end{tabular}


supporting information

$\begin{array}{lllll}\mathrm{H} 19 & 0.5810 & 0.3465 & 0.5647 & 0.052^{*} \\ \mathrm{C} 20 & 0.5561(3) & 0.51389(15) & 0.58591(15) & 0.0408(8) \\ \mathrm{H} 20 & 0.5861 & 0.5626 & 0.5929 & 0.049^{*} \\ \mathrm{C} 21 & 0.7878(3) & 0.45277(14) & 0.58206(14) & 0.0326(7) \\ \mathrm{C} 22 & 0.8581(3) & 0.38678(15) & 0.58217(15) & 0.0401(8) \\ \mathrm{H} 22 & 0.8129 & 0.3411 & 0.5804 & 0.048^{*} \\ \mathrm{C} 23 & 0.9995(3) & 0.39140(15) & 0.58510(16) & 0.0443(8) \\ \mathrm{H} 23 & 1.0520 & 0.3480 & 0.5854 & 0.053^{*} \\ \mathrm{C} 24 & 1.0638(3) & 0.45981(15) & 0.58760(15) & 0.0413(8) \\ \mathrm{H} 24 & 1.1592 & 0.4639 & 0.5896 & 0.050^{*} \\ \mathrm{C} 25 & 0.9809(3) & 0.52181(14) & 0.58709(15) & 0.0358(7) \\ \mathrm{C} 26 & 0.9662(3) & 0.66025(16) & 0.58895(18) & 0.0559(10) \\ \mathrm{H} 26 & 0.8709 & 0.6639 & 0.5901 & 0.067^{*} \\ \mathrm{C} 27 & 1.1704(3) & 0.61615(16) & 0.58718(16) & 0.0482(8) \\ \mathrm{H} 27 & 1.2446 & 0.5835 & 0.5867 & 0.058^{*}\end{array}$

Atomic displacement parameters $\left(\AA^{2}\right)$

\begin{tabular}{|c|c|c|c|c|c|c|}
\hline & $U^{11}$ & $U^{22}$ & $U^{33}$ & $U^{12}$ & $U^{13}$ & $U^{23}$ \\
\hline $\mathrm{O} 1$ & $0.0398(13)$ & $0.0492(13)$ & $0.109(2)$ & $0.0011(10)$ & $-0.0098(13)$ & $-0.0046(14)$ \\
\hline $\mathrm{O} 2$ & $0.0740(18)$ & $0.0638(16)$ & $0.109(2)$ & $0.0206(13)$ & $0.0131(17)$ & $0.0014(18)$ \\
\hline $\mathrm{O} 3$ & $0.0645(17)$ & $0.0626(16)$ & $0.106(3)$ & $-0.0161(13)$ & $-0.0046(15)$ & $0.0018(15)$ \\
\hline $\mathrm{O} 4$ & $0.0360(13)$ & $0.0498(13)$ & $0.118(2)$ & $-0.0005(10)$ & $0.0105(13)$ & $-0.0040(15)$ \\
\hline O5 & $0.0698(18)$ & $0.0691(17)$ & $0.107(2)$ & $0.0234(14)$ & $0.0024(16)$ & $0.0062(18)$ \\
\hline O6 & $0.0393(13)$ & $0.0498(13)$ & $0.129(2)$ & $0.0028(10)$ & $-0.0090(14)$ & $-0.0066(16)$ \\
\hline N1 & $0.0353(15)$ & $0.0441(15)$ & $0.061(2)$ & $-0.0023(12)$ & $0.0029(13)$ & $-0.0056(13)$ \\
\hline N2 & $0.0373(15)$ & $0.0420(15)$ & $0.062(2)$ & $0.0040(11)$ & $-0.0004(13)$ & $-0.0034(13)$ \\
\hline N3 & $0.0310(13)$ & $0.0298(12)$ & $0.0484(18)$ & $0.0015(11)$ & $0.0026(11)$ & $-0.0044(11)$ \\
\hline N4 & $0.0298(13)$ & $0.0309(12)$ & $0.0412(17)$ & $-0.0004(10)$ & $0.0012(11)$ & $0.0022(11)$ \\
\hline N5 & $0.0317(13)$ & $0.0319(13)$ & $0.0520(18)$ & $-0.0016(10)$ & $-0.0013(12)$ & $0.0042(11)$ \\
\hline N6 & $0.0444(16)$ & $0.0377(14)$ & $0.080(2)$ & $-0.0068(12)$ & $0.0027(14)$ & $0.0004(14)$ \\
\hline N7 & $0.0389(16)$ & $0.0442(16)$ & $0.090(2)$ & $-0.0045(12)$ & $0.0004(15)$ & $0.0004(15)$ \\
\hline N8 & $0.0336(14)$ & $0.0402(14)$ & $0.068(2)$ & $-0.0001(11)$ & $0.0041(13)$ & $-0.0020(13)$ \\
\hline N9 & $0.0315(14)$ & $0.0442(15)$ & 0.0587 (19) & $0.0024(11)$ & $0.0030(12)$ & $0.0008(13)$ \\
\hline N10 & $0.0274(12)$ & $0.0296(12)$ & $0.0487(17)$ & $0.0001(10)$ & $0.0022(11)$ & $-0.0013(11)$ \\
\hline N11 & $0.0267(12)$ & $0.0322(12)$ & $0.0421(16)$ & $0.0026(10)$ & $-0.0008(10)$ & $-0.0003(11)$ \\
\hline $\mathrm{N} 12$ & $0.0276(12)$ & $0.0301(12)$ & $0.0509(18)$ & $-0.0025(10)$ & $0.0005(11)$ & $-0.0002(12)$ \\
\hline N13 & $0.0429(15)$ & $0.0376(14)$ & $0.067(2)$ & $-0.0027(12)$ & $0.0027(13)$ & $0.0070(13)$ \\
\hline N14 & $0.0407(15)$ & $0.0441(15)$ & $0.058(2)$ & $-0.0089(12)$ & $0.0003(13)$ & $0.0015(13)$ \\
\hline N15 & $0.0320(14)$ & $0.0385(14)$ & $0.065(2)$ & $-0.0043(11)$ & $-0.0005(13)$ & $0.0064(13)$ \\
\hline N16 & $0.0321(14)$ & $0.0437(15)$ & $0.058(2)$ & $0.0009(11)$ & $0.0023(12)$ & $0.0012(13)$ \\
\hline N17 & $0.0287(12)$ & $0.0302(12)$ & $0.0429(17)$ & $-0.0002(10)$ & $0.0000(11)$ & $0.0018(11)$ \\
\hline N18 & $0.0299(13)$ & $0.0345(13)$ & $0.0423(17)$ & $0.0015(10)$ & $0.0019(11)$ & $0.0015(11)$ \\
\hline N19 & $0.0303(13)$ & $0.0348(13)$ & $0.0522(18)$ & $-0.0024(11)$ & $-0.0019(12)$ & $-0.0010(12)$ \\
\hline $\mathrm{N} 20$ & 0.0495 (17) & $0.0432(16)$ & $0.095(3)$ & $-0.0043(14)$ & $0.0004(16)$ & $-0.0010(15)$ \\
\hline $\mathrm{N} 21$ & $0.0435(16)$ & $0.0473(16)$ & $0.077(2)$ & $-0.0086(13)$ & $-0.0016(14)$ & $0.0052(15)$ \\
\hline $\mathrm{C} 1$ & $0.0402(17)$ & $0.0327(16)$ & $0.057(2)$ & $-0.0018(14)$ & $0.0015(15)$ & $-0.0022(15)$ \\
\hline $\mathrm{C} 2$ & $0.0384(17)$ & $0.0344(16)$ & $0.053(2)$ & $0.0030(14)$ & $-0.0020(15)$ & $-0.0015(15)$ \\
\hline
\end{tabular}


supporting information

\begin{tabular}{lllllll} 
C3 & $0.0323(16)$ & $0.0302(15)$ & $0.043(2)$ & $-0.0007(12)$ & $0.0024(14)$ & $0.0019(13)$ \\
C4 & $0.0411(18)$ & $0.0290(16)$ & $0.075(3)$ & $0.0002(13)$ & $0.0042(17)$ & $-0.0064(15)$ \\
C5 & $0.0389(18)$ & $0.0368(17)$ & $0.087(3)$ & $0.0104(14)$ & $0.0042(17)$ & $-0.0047(17)$ \\
C6 & $0.0314(16)$ & $0.0366(17)$ & $0.073(3)$ & $0.0040(13)$ & $0.0050(16)$ & $-0.0012(16)$ \\
C7 & $0.0360(16)$ & $0.0276(14)$ & $0.039(2)$ & $-0.0016(12)$ & $0.0019(14)$ & $0.0005(13)$ \\
C8 & $0.0403(18)$ & $0.0357(17)$ & $0.063(2)$ & $0.0020(14)$ & $-0.0007(16)$ & $0.0036(16)$ \\
C9 & $0.0364(17)$ & $0.0409(18)$ & $0.068(3)$ & $-0.0007(14)$ & $-0.0032(16)$ & $0.0034(16)$ \\
C10 & $0.0327(17)$ & $0.0329(16)$ & $0.072(3)$ & $0.0004(13)$ & $0.0010(16)$ & $-0.0039(15)$ \\
C11 & $0.0348(17)$ & $0.0376(16)$ & $0.047(2)$ & $0.0049(13)$ & $0.0031(14)$ & $0.0011(14)$ \\
C12 & $0.0251(14)$ & $0.0335(15)$ & $0.041(2)$ & $0.0015(12)$ & $-0.0003(13)$ & $-0.0058(13)$ \\
C13 & $0.0380(17)$ & $0.0311(15)$ & $0.066(2)$ & $0.0011(13)$ & $-0.0020(16)$ & $-0.0004(15)$ \\
C14 & $0.0388(18)$ & $0.0360(17)$ & $0.071(3)$ & $0.0123(13)$ & $-0.0063(16)$ & $-0.0029(16)$ \\
C15 & $0.0273(15)$ & $0.0428(17)$ & $0.059(2)$ & $0.0034(13)$ & $-0.0029(14)$ & $-0.0045(16)$ \\
C16 & $0.0302(16)$ & $0.0332(15)$ & $0.041(2)$ & $0.0008(12)$ & $-0.0011(13)$ & $-0.0064(14)$ \\
C17 & $0.0350(16)$ & $0.0382(17)$ & $0.058(2)$ & $0.0014(14)$ & $0.0019(15)$ & $0.0013(15)$ \\
C18 & $0.0334(17)$ & $0.0452(18)$ & $0.049(2)$ & $-0.0012(14)$ & $0.0000(15)$ & $-0.0014(16)$ \\
C19 & $0.0383(17)$ & $0.0314(15)$ & $0.061(2)$ & $-0.0043(13)$ & $-0.0044(15)$ & $-0.0010(15)$ \\
C20 & $0.0377(17)$ & $0.0342(16)$ & $0.051(2)$ & $0.0031(14)$ & $0.0024(15)$ & $-0.0023(15)$ \\
C21 & $0.0273(15)$ & $0.0339(15)$ & $0.0368(19)$ & $-0.0003(12)$ & $0.0017(13)$ & $0.0016(13)$ \\
C22 & $0.0384(17)$ & $0.0305(15)$ & $0.051(2)$ & $-0.0015(13)$ & $0.0012(15)$ & $-0.0026(14)$ \\
C23 & $0.0401(18)$ & $0.0321(16)$ & $0.061(2)$ & $0.0073(13)$ & $0.0020(16)$ & $-0.0006(15)$ \\
C24 & $0.0238(15)$ & $0.0435(17)$ & $0.057(2)$ & $0.0048(13)$ & $-0.0017(14)$ & $-0.0008(15)$ \\
C25 & $0.0342(16)$ & $0.0325(15)$ & $0.041(2)$ & $-0.0018(13)$ & $0.0011(14)$ & $-0.0011(14)$ \\
C26 & $0.0396(18)$ & $0.0373(18)$ & $0.091(3)$ & $-0.0021(15)$ & $-0.0006(18)$ & $0.0027(18)$ \\
C27 & $0.0360(18)$ & $0.0460(19)$ & $0.062(2)$ & $-0.0037(14)$ & $-0.0041(16)$ & $0.0043(16)$ \\
& & & & & & \\
\hline
\end{tabular}

Geometric parameters $\left(\AA,{ }^{\circ}\right)$

\begin{tabular}{llll}
\hline $\mathrm{O} 1-\mathrm{H} 1 \mathrm{~A}$ & $0.857(10)$ & $\mathrm{N} 17-\mathrm{C} 20$ & $1.363(3)$ \\
$\mathrm{O} 1-\mathrm{H} 1 \mathrm{~B}$ & $0.859(10)$ & $\mathrm{N} 17-\mathrm{C} 21$ & $1.425(3)$ \\
$\mathrm{O} 2-\mathrm{H} 2 \mathrm{~A}$ & $0.868(10)$ & $\mathrm{N} 18-\mathrm{C} 21$ & $1.325(3)$ \\
$\mathrm{O} 2-\mathrm{H} 2 \mathrm{~B}$ & $0.858(10)$ & $\mathrm{N} 18-\mathrm{C} 25$ & $1.329(3)$ \\
$\mathrm{O} 3-\mathrm{H} 3 \mathrm{~A}$ & $0.854(10)$ & $\mathrm{N} 19-\mathrm{C} 27$ & $1.357(3)$ \\
$\mathrm{O} 3-\mathrm{H} 3 \mathrm{~B}$ & $0.856(10)$ & $\mathrm{N} 19-\mathrm{C} 26$ & $1.359(3)$ \\
$\mathrm{O} 4-\mathrm{H} 4 \mathrm{~A}$ & $0.861(10)$ & $\mathrm{N} 19-\mathrm{C} 25$ & $1.414(3)$ \\
$\mathrm{O} 4-\mathrm{H} 4 \mathrm{~B}$ & $0.856(10)$ & $\mathrm{N} 20-\mathrm{C} 26$ & $1.294(3)$ \\
$\mathrm{O} 5-\mathrm{H} 5 \mathrm{~A}$ & $0.868(10)$ & $\mathrm{N} 20-\mathrm{N} 21$ & $1.390(3)$ \\
$\mathrm{O} 5-\mathrm{H} 5 \mathrm{~B}$ & $0.861(10)$ & $\mathrm{N} 21-\mathrm{C} 27$ & 0.9300 \\
$\mathrm{O} 6-\mathrm{H} 6 \mathrm{~A}$ & $0.861(10)$ & $\mathrm{C} 1-\mathrm{H} 1$ & 0.9300 \\
$\mathrm{O} 6-\mathrm{H} 6 \mathrm{~B}$ & $0.858(10)$ & $\mathrm{C} 2-\mathrm{H} 2$ & $1.373(3)$ \\
$\mathrm{N} 1-\mathrm{C} 1$ & $1.288(3)$ & $\mathrm{C} 3-\mathrm{C} 4$ & $1.384(4)$ \\
$\mathrm{N} 1-\mathrm{N} 2$ & $1.393(3)$ & $\mathrm{C} 4-\mathrm{C} 5$ & 0.9300 \\
$\mathrm{~N} 2-\mathrm{C} 2$ & $1.298(3)$ & $\mathrm{C} 4-\mathrm{H} 4$ & $1.366(4)$ \\
$\mathrm{N} 3-\mathrm{C} 1$ & $1.360(3)$ & $\mathrm{C} 5-\mathrm{C} 6$ & 0.9300 \\
$\mathrm{~N} 3-\mathrm{C} 2$ & $1.361(3)$ & $\mathrm{C} 5-\mathrm{H} 5$ & $1.379(3)$ \\
$\mathrm{N} 3-\mathrm{C} 3$ & $1.413(3)$ & $\mathrm{C} 6-\mathrm{C} 7$ & 0.9300 \\
$\mathrm{~N} 4-\mathrm{C} 7$ & $1.323(3)$ & $\mathrm{C} 6-\mathrm{H} 6$ & 0.9300 \\
$\mathrm{~N} 4-\mathrm{C} 3$ & $1.329(3)$ & $\mathrm{C} 8-\mathrm{H} 8$ &
\end{tabular}




\begin{tabular}{|c|c|c|c|}
\hline $\mathrm{N} 5-\mathrm{C} 9$ & $1.359(3)$ & $\mathrm{C} 9-\mathrm{H} 9$ & 0.9300 \\
\hline $\mathrm{N} 5-\mathrm{C} 8$ & $1.359(3)$ & $\mathrm{C} 10-\mathrm{H} 10$ & 0.9300 \\
\hline $\mathrm{N} 5-\mathrm{C} 7$ & $1.421(3)$ & $\mathrm{C} 11-\mathrm{H} 11$ & 0.9300 \\
\hline $\mathrm{N} 6-\mathrm{C} 8$ & $1.298(3)$ & $\mathrm{C} 12-\mathrm{C} 13$ & $1.377(3)$ \\
\hline N6-N7 & $1.386(3)$ & $\mathrm{C} 13-\mathrm{C} 14$ & $1.371(4)$ \\
\hline $\mathrm{N} 7-\mathrm{C} 9$ & $1.294(3)$ & $\mathrm{C} 13-\mathrm{H} 13$ & 0.9300 \\
\hline $\mathrm{N} 8-\mathrm{C} 10$ & $1.295(3)$ & $\mathrm{C} 14-\mathrm{C} 15$ & $1.371(4)$ \\
\hline N8-N9 & $1.390(3)$ & $\mathrm{C} 14-\mathrm{H} 14$ & 0.9300 \\
\hline N9-C11 & $1.300(3)$ & $\mathrm{C} 15-\mathrm{C} 16$ & $1.379(3)$ \\
\hline $\mathrm{N} 10-\mathrm{C} 10$ & $1.354(3)$ & C15-H15 & 0.9300 \\
\hline $\mathrm{N} 10-\mathrm{C} 11$ & $1.364(3)$ & $\mathrm{C} 17-\mathrm{H} 17$ & 0.9300 \\
\hline $\mathrm{N} 10-\mathrm{C} 12$ & $1.420(3)$ & $\mathrm{C} 18-\mathrm{H} 18$ & 0.9300 \\
\hline $\mathrm{N} 11-\mathrm{C} 16$ & $1.318(3)$ & $\mathrm{C} 19-\mathrm{H} 19$ & 0.9300 \\
\hline $\mathrm{N} 11-\mathrm{C} 12$ & $1.325(3)$ & $\mathrm{C} 20-\mathrm{H} 20$ & 0.9300 \\
\hline $\mathrm{N} 12-\mathrm{C} 18$ & $1.357(3)$ & $\mathrm{C} 21-\mathrm{C} 22$ & $1.366(3)$ \\
\hline $\mathrm{N} 12-\mathrm{C} 17$ & $1.357(3)$ & $\mathrm{C} 22-\mathrm{C} 23$ & $1.377(4)$ \\
\hline $\mathrm{N} 12-\mathrm{C} 16$ & $1.422(3)$ & $\mathrm{C} 22-\mathrm{H} 22$ & 0.9300 \\
\hline $\mathrm{N} 13-\mathrm{C} 17$ & $1.296(3)$ & $\mathrm{C} 23-\mathrm{C} 24$ & $1.376(4)$ \\
\hline N13-N14 & $1.385(3)$ & $\mathrm{C} 23-\mathrm{H} 23$ & 0.9300 \\
\hline $\mathrm{N} 14-\mathrm{C} 18$ & $1.294(3)$ & $\mathrm{C} 24-\mathrm{C} 25$ & $1.373(3)$ \\
\hline $\mathrm{N} 15-\mathrm{C} 19$ & $1.295(3)$ & $\mathrm{C} 24-\mathrm{H} 24$ & 0.9300 \\
\hline N15-N16 & $1.389(3)$ & $\mathrm{C} 26-\mathrm{H} 26$ & 0.9300 \\
\hline $\mathrm{N} 16-\mathrm{C} 20$ & $1.291(3)$ & $\mathrm{C} 27-\mathrm{H} 27$ & 0.9300 \\
\hline N17-C19 & $1.356(3)$ & & \\
\hline $\mathrm{H} 1 \mathrm{~A}-\mathrm{O} 1-\mathrm{H} 1 \mathrm{~B}$ & $108.9(16)$ & $\mathrm{N} 6-\mathrm{C} 8-\mathrm{N} 5$ & $110.9(3)$ \\
\hline $\mathrm{H} 2 \mathrm{~A}-\mathrm{O} 2-\mathrm{H} 2 \mathrm{~B}$ & $107.5(16)$ & $\mathrm{N} 6-\mathrm{C} 8-\mathrm{H} 8$ & 124.5 \\
\hline $\mathrm{H} 3 \mathrm{~A}-\mathrm{O} 3-\mathrm{H} 3 \mathrm{~B}$ & $109.4(16)$ & $\mathrm{N} 5-\mathrm{C} 8-\mathrm{H} 8$ & 124.5 \\
\hline $\mathrm{H} 4 \mathrm{~A}-\mathrm{O} 4-\mathrm{H} 4 \mathrm{~B}$ & $108.5(16)$ & $\mathrm{N} 7-\mathrm{C} 9-\mathrm{N} 5$ & $109.9(2)$ \\
\hline $\mathrm{H} 5 \mathrm{~A}-\mathrm{O} 5-\mathrm{H} 5 \mathrm{~B}$ & $107.8(16)$ & $\mathrm{N} 7-\mathrm{C} 9-\mathrm{H} 9$ & 125.0 \\
\hline $\mathrm{H} 6 \mathrm{~A}-\mathrm{O} 6-\mathrm{H} 6 \mathrm{~B}$ & $109.3(16)$ & $\mathrm{N} 5-\mathrm{C} 9-\mathrm{H} 9$ & 125.0 \\
\hline $\mathrm{C} 1-\mathrm{N} 1-\mathrm{N} 2$ & $107.4(2)$ & $\mathrm{N} 8-\mathrm{C} 10-\mathrm{N} 10$ & $110.9(2)$ \\
\hline $\mathrm{C} 2-\mathrm{N} 2-\mathrm{N} 1$ & $106.4(2)$ & $\mathrm{N} 8-\mathrm{C} 10-\mathrm{H} 10$ & 124.6 \\
\hline $\mathrm{C} 1-\mathrm{N} 3-\mathrm{C} 2$ & $103.9(2)$ & $\mathrm{N} 10-\mathrm{C} 10-\mathrm{H} 10$ & 124.6 \\
\hline $\mathrm{C} 1-\mathrm{N} 3-\mathrm{C} 3$ & $128.4(2)$ & $\mathrm{N} 9-\mathrm{C} 11-\mathrm{N} 10$ & $111.0(2)$ \\
\hline $\mathrm{C} 2-\mathrm{N} 3-\mathrm{C} 3$ & $127.6(2)$ & N9-C11-H11 & 124.5 \\
\hline $\mathrm{C} 7-\mathrm{N} 4-\mathrm{C} 3$ & $116.5(2)$ & $\mathrm{N} 10-\mathrm{C} 11-\mathrm{H} 11$ & 124.5 \\
\hline $\mathrm{C} 9-\mathrm{N} 5-\mathrm{C} 8$ & $104.7(2)$ & $\mathrm{N} 11-\mathrm{C} 12-\mathrm{C} 13$ & $124.7(2)$ \\
\hline $\mathrm{C} 9-\mathrm{N} 5-\mathrm{C} 7$ & $128.3(2)$ & $\mathrm{N} 11-\mathrm{C} 12-\mathrm{N} 10$ & $114.0(2)$ \\
\hline $\mathrm{C} 8-\mathrm{N} 5-\mathrm{C} 7$ & $127.0(2)$ & $\mathrm{C} 13-\mathrm{C} 12-\mathrm{N} 10$ & $121.3(2)$ \\
\hline $\mathrm{C} 8-\mathrm{N} 6-\mathrm{N} 7$ & $106.3(2)$ & $\mathrm{C} 14-\mathrm{C} 13-\mathrm{C} 12$ & $116.7(3)$ \\
\hline $\mathrm{C} 9-\mathrm{N} 7-\mathrm{N} 6$ & $108.1(2)$ & $\mathrm{C} 14-\mathrm{C} 13-\mathrm{H} 13$ & 121.7 \\
\hline $\mathrm{C} 10-\mathrm{N} 8-\mathrm{N} 9$ & $107.5(2)$ & $\mathrm{C} 12-\mathrm{C} 13-\mathrm{H} 13$ & 121.7 \\
\hline $\mathrm{C} 11-\mathrm{N} 9-\mathrm{N} 8$ & $106.4(2)$ & $\mathrm{C} 13-\mathrm{C} 14-\mathrm{C} 15$ & $120.7(3)$ \\
\hline $\mathrm{C} 10-\mathrm{N} 10-\mathrm{C} 11$ & $104.2(2)$ & $\mathrm{C} 13-\mathrm{C} 14-\mathrm{H} 14$ & 119.7 \\
\hline $\mathrm{C} 10-\mathrm{N} 10-\mathrm{C} 12$ & $128.4(2)$ & $\mathrm{C} 15-\mathrm{C} 14-\mathrm{H} 14$ & 119.7 \\
\hline $\mathrm{C} 11-\mathrm{N} 10-\mathrm{C} 12$ & $127.3(2)$ & $\mathrm{C} 14-\mathrm{C} 15-\mathrm{C} 16$ & $117.0(3)$ \\
\hline $\mathrm{C} 16-\mathrm{N} 11-\mathrm{C} 12$ & $116.5(2)$ & $\mathrm{C} 14-\mathrm{C} 15-\mathrm{H} 15$ & 121.5 \\
\hline
\end{tabular}




\begin{tabular}{|c|c|c|c|}
\hline $\mathrm{C} 18-\mathrm{N} 12-\mathrm{C} 17$ & $104.4(2)$ & $\mathrm{C} 16-\mathrm{C} 15-\mathrm{H} 15$ & 121.5 \\
\hline $\mathrm{C} 18-\mathrm{N} 12-\mathrm{C} 16$ & $128.9(2)$ & $\mathrm{N} 11-\mathrm{C} 16-\mathrm{C} 15$ & $124.5(2)$ \\
\hline $\mathrm{C} 17-\mathrm{N} 12-\mathrm{C} 16$ & $126.7(2)$ & $\mathrm{N} 11-\mathrm{C} 16-\mathrm{N} 12$ & $113.9(2)$ \\
\hline C17-N13-N14 & $106.9(2)$ & $\mathrm{C} 15-\mathrm{C} 16-\mathrm{N} 12$ & $121.6(2)$ \\
\hline $\mathrm{C} 18-\mathrm{N} 14-\mathrm{N} 13$ & $107.2(2)$ & $\mathrm{N} 13-\mathrm{C} 17-\mathrm{N} 12$ & $110.8(2)$ \\
\hline $\mathrm{C} 19-\mathrm{N} 15-\mathrm{N} 16$ & $106.8(2)$ & $\mathrm{N} 13-\mathrm{C} 17-\mathrm{H} 17$ & 124.6 \\
\hline $\mathrm{C} 20-\mathrm{N} 16-\mathrm{N} 15$ & $107.0(2)$ & $\mathrm{N} 12-\mathrm{C} 17-\mathrm{H} 17$ & 124.6 \\
\hline $\mathrm{C} 19-\mathrm{N} 17-\mathrm{C} 20$ & $103.8(2)$ & $\mathrm{N} 14-\mathrm{C} 18-\mathrm{N} 12$ & $110.7(2)$ \\
\hline $\mathrm{C} 19-\mathrm{N} 17-\mathrm{C} 21$ & $128.2(2)$ & $\mathrm{N} 14-\mathrm{C} 18-\mathrm{H} 18$ & 124.6 \\
\hline $\mathrm{C} 20-\mathrm{N} 17-\mathrm{C} 21$ & $128.0(2)$ & $\mathrm{N} 12-\mathrm{C} 18-\mathrm{H} 18$ & 124.6 \\
\hline $\mathrm{C} 21-\mathrm{N} 18-\mathrm{C} 25$ & $115.9(2)$ & $\mathrm{N} 15-\mathrm{C} 19-\mathrm{N} 17$ & $111.4(2)$ \\
\hline $\mathrm{C} 27-\mathrm{N} 19-\mathrm{C} 26$ & $103.8(2)$ & N15-C19-H19 & 124.3 \\
\hline $\mathrm{C} 27-\mathrm{N} 19-\mathrm{C} 25$ & $128.9(2)$ & N17-C19-H19 & 124.3 \\
\hline $\mathrm{C} 26-\mathrm{N} 19-\mathrm{C} 25$ & $127.3(2)$ & $\mathrm{N} 16-\mathrm{C} 20-\mathrm{N} 17$ & $111.2(2)$ \\
\hline $\mathrm{C} 26-\mathrm{N} 20-\mathrm{N} 21$ & $106.1(2)$ & $\mathrm{N} 16-\mathrm{C} 20-\mathrm{H} 20$ & 124.4 \\
\hline $\mathrm{C} 27-\mathrm{N} 21-\mathrm{N} 20$ & $107.6(2)$ & $\mathrm{N} 17-\mathrm{C} 20-\mathrm{H} 20$ & 124.4 \\
\hline $\mathrm{N} 1-\mathrm{C} 1-\mathrm{N} 3$ & $111.1(2)$ & $\mathrm{N} 18-\mathrm{C} 21-\mathrm{C} 22$ & $125.5(2)$ \\
\hline $\mathrm{N} 1-\mathrm{C} 1-\mathrm{H} 1$ & 124.4 & $\mathrm{~N} 18-\mathrm{C} 21-\mathrm{N} 17$ & $113.7(2)$ \\
\hline $\mathrm{N} 3-\mathrm{C} 1-\mathrm{H} 1$ & 124.4 & $\mathrm{C} 22-\mathrm{C} 21-\mathrm{N} 17$ & $120.9(2)$ \\
\hline $\mathrm{N} 2-\mathrm{C} 2-\mathrm{N} 3$ & $111.2(2)$ & $\mathrm{C} 21-\mathrm{C} 22-\mathrm{C} 23$ & $116.6(3)$ \\
\hline $\mathrm{N} 2-\mathrm{C} 2-\mathrm{H} 2$ & 124.4 & $\mathrm{C} 21-\mathrm{C} 22-\mathrm{H} 22$ & 121.7 \\
\hline $\mathrm{N} 3-\mathrm{C} 2-\mathrm{H} 2$ & 124.4 & $\mathrm{C} 23-\mathrm{C} 22-\mathrm{H} 22$ & 121.7 \\
\hline $\mathrm{N} 4-\mathrm{C} 3-\mathrm{C} 4$ & $124.6(2)$ & $\mathrm{C} 24-\mathrm{C} 23-\mathrm{C} 22$ & $120.5(3)$ \\
\hline $\mathrm{N} 4-\mathrm{C} 3-\mathrm{N} 3$ & $114.1(2)$ & $\mathrm{C} 24-\mathrm{C} 23-\mathrm{H} 23$ & 119.8 \\
\hline $\mathrm{C} 4-\mathrm{C} 3-\mathrm{N} 3$ & $121.3(2)$ & $\mathrm{C} 22-\mathrm{C} 23-\mathrm{H} 23$ & 119.8 \\
\hline $\mathrm{C} 3-\mathrm{C} 4-\mathrm{C} 5$ & $116.8(3)$ & $\mathrm{C} 25-\mathrm{C} 24-\mathrm{C} 23$ & $117.0(2)$ \\
\hline $\mathrm{C} 3-\mathrm{C} 4-\mathrm{H} 4$ & 121.6 & $\mathrm{C} 25-\mathrm{C} 24-\mathrm{H} 24$ & 121.5 \\
\hline $\mathrm{C} 5-\mathrm{C} 4-\mathrm{H} 4$ & 121.6 & $\mathrm{C} 23-\mathrm{C} 24-\mathrm{H} 24$ & 121.5 \\
\hline $\mathrm{C} 6-\mathrm{C} 5-\mathrm{C} 4$ & $120.6(3)$ & $\mathrm{N} 18-\mathrm{C} 25-\mathrm{C} 24$ & $124.6(2)$ \\
\hline $\mathrm{C} 6-\mathrm{C} 5-\mathrm{H} 5$ & 119.7 & $\mathrm{~N} 18-\mathrm{C} 25-\mathrm{N} 19$ & $113.9(2)$ \\
\hline $\mathrm{C} 4-\mathrm{C} 5-\mathrm{H} 5$ & 119.7 & $\mathrm{C} 24-\mathrm{C} 25-\mathrm{N} 19$ & $121.6(2)$ \\
\hline $\mathrm{C} 5-\mathrm{C} 6-\mathrm{C} 7$ & $117.1(3)$ & $\mathrm{N} 20-\mathrm{C} 26-\mathrm{N} 19$ & $111.6(3)$ \\
\hline $\mathrm{C} 5-\mathrm{C} 6-\mathrm{H} 6$ & 121.4 & $\mathrm{~N} 20-\mathrm{C} 26-\mathrm{H} 26$ & 124.2 \\
\hline $\mathrm{C} 7-\mathrm{C} 6-\mathrm{H} 6$ & 121.4 & $\mathrm{~N} 19-\mathrm{C} 26-\mathrm{H} 26$ & 124.2 \\
\hline $\mathrm{N} 4-\mathrm{C} 7-\mathrm{C} 6$ & $124.5(2)$ & $\mathrm{N} 21-\mathrm{C} 27-\mathrm{N} 19$ & $111.0(3)$ \\
\hline $\mathrm{N} 4-\mathrm{C} 7-\mathrm{N} 5$ & $114.0(2)$ & $\mathrm{N} 21-\mathrm{C} 27-\mathrm{H} 27$ & 124.5 \\
\hline $\mathrm{C} 6-\mathrm{C} 7-\mathrm{N} 5$ & $121.5(2)$ & $\mathrm{N} 19-\mathrm{C} 27-\mathrm{H} 27$ & 124.5 \\
\hline $\mathrm{C} 1-\mathrm{N} 1-\mathrm{N} 2-\mathrm{C} 2$ & $-0.4(3)$ & $\mathrm{N} 11-\mathrm{C} 12-\mathrm{C} 13-\mathrm{C} 14$ & $0.0(5)$ \\
\hline $\mathrm{C} 8-\mathrm{N} 6-\mathrm{N} 7-\mathrm{C} 9$ & $0.0(4)$ & $\mathrm{N} 10-\mathrm{C} 12-\mathrm{C} 13-\mathrm{C} 14$ & $-179.9(3)$ \\
\hline $\mathrm{C} 10-\mathrm{N} 8-\mathrm{N} 9-\mathrm{C} 11$ & $-0.2(3)$ & $\mathrm{C} 12-\mathrm{C} 13-\mathrm{C} 14-\mathrm{C} 15$ & $-0.5(5)$ \\
\hline $\mathrm{C} 17-\mathrm{N} 13-\mathrm{N} 14-\mathrm{C} 18$ & $0.0(3)$ & $\mathrm{C} 13-\mathrm{C} 14-\mathrm{C} 15-\mathrm{C} 16$ & $1.2(5)$ \\
\hline $\mathrm{C} 19-\mathrm{N} 15-\mathrm{N} 16-\mathrm{C} 20$ & $0.2(3)$ & $\mathrm{C} 12-\mathrm{N} 11-\mathrm{C} 16-\mathrm{C} 15$ & $1.1(4)$ \\
\hline $\mathrm{C} 26-\mathrm{N} 20-\mathrm{N} 21-\mathrm{C} 27$ & $0.4(4)$ & $\mathrm{C} 12-\mathrm{N} 11-\mathrm{C} 16-\mathrm{N} 12$ & $179.6(2)$ \\
\hline $\mathrm{N} 2-\mathrm{N} 1-\mathrm{C} 1-\mathrm{N} 3$ & $-0.3(3)$ & $\mathrm{C} 14-\mathrm{C} 15-\mathrm{C} 16-\mathrm{N} 11$ & $-1.6(5)$ \\
\hline $\mathrm{C} 2-\mathrm{N} 3-\mathrm{C} 1-\mathrm{N} 1$ & $0.8(3)$ & $\mathrm{C} 14-\mathrm{C} 15-\mathrm{C} 16-\mathrm{N} 12$ & $180.0(3)$ \\
\hline $\mathrm{C} 3-\mathrm{N} 3-\mathrm{C} 1-\mathrm{N} 1$ & $-178.3(3)$ & $\mathrm{C} 18-\mathrm{N} 12-\mathrm{C} 16-\mathrm{N} 11$ & $-171.5(3)$ \\
\hline $\mathrm{N} 1-\mathrm{N} 2-\mathrm{C} 2-\mathrm{N} 3$ & $0.9(3)$ & $\mathrm{C} 17-\mathrm{N} 12-\mathrm{C} 16-\mathrm{N} 11$ & $7.8(4)$ \\
\hline
\end{tabular}




\begin{tabular}{|c|c|}
\hline $\mathrm{C} 1-\mathrm{N} 3-\mathrm{C} 2-\mathrm{N} 2$ & $-1.1(3)$ \\
\hline $\mathrm{C} 3-\mathrm{N} 3-\mathrm{C} 2-\mathrm{N} 2$ & $178.1(3)$ \\
\hline $\mathrm{C} 7-\mathrm{N} 4-\mathrm{C} 3-\mathrm{C} 4$ & $0.4(4)$ \\
\hline $\mathrm{C} 7-\mathrm{N} 4-\mathrm{C} 3-\mathrm{N} 3$ & $-179.3(2)$ \\
\hline $\mathrm{C} 1-\mathrm{N} 3-\mathrm{C} 3-\mathrm{N} 4$ & $-176.2(3)$ \\
\hline $\mathrm{C} 2-\mathrm{N} 3-\mathrm{C} 3-\mathrm{N} 4$ & $4.9(4)$ \\
\hline $\mathrm{C} 1-\mathrm{N} 3-\mathrm{C} 3-\mathrm{C} 4$ & $4.2(5)$ \\
\hline $\mathrm{C} 2-\mathrm{N} 3-\mathrm{C} 3-\mathrm{C} 4$ & $-174.7(3)$ \\
\hline $\mathrm{N} 4-\mathrm{C} 3-\mathrm{C} 4-\mathrm{C} 5$ & $-0.1(5)$ \\
\hline $\mathrm{N} 3-\mathrm{C} 3-\mathrm{C} 4-\mathrm{C} 5$ & $179.5(3)$ \\
\hline $\mathrm{C} 3-\mathrm{C} 4-\mathrm{C} 5-\mathrm{C} 6$ & $0.1(5)$ \\
\hline $\mathrm{C} 4-\mathrm{C} 5-\mathrm{C} 6-\mathrm{C} 7$ & $-0.4(5)$ \\
\hline $\mathrm{C} 3-\mathrm{N} 4-\mathrm{C} 7-\mathrm{C} 6$ & $-0.7(4)$ \\
\hline $\mathrm{C} 3-\mathrm{N} 4-\mathrm{C} 7-\mathrm{N} 5$ & $179.1(2)$ \\
\hline $\mathrm{C} 5-\mathrm{C} 6-\mathrm{C} 7-\mathrm{N} 4$ & $0.7(5)$ \\
\hline $\mathrm{C} 5-\mathrm{C} 6-\mathrm{C} 7-\mathrm{N} 5$ & $-179.1(3)$ \\
\hline $\mathrm{C} 9-\mathrm{N} 5-\mathrm{C} 7-\mathrm{N} 4$ & $-178.5(3)$ \\
\hline $\mathrm{C} 8-\mathrm{N} 5-\mathrm{C} 7-\mathrm{N} 4$ & $0.6(4)$ \\
\hline $\mathrm{C} 9-\mathrm{N} 5-\mathrm{C} 7-\mathrm{C} 6$ & $1.3(5)$ \\
\hline $\mathrm{C} 8-\mathrm{N} 5-\mathrm{C} 7-\mathrm{C} 6$ & $-179.6(3)$ \\
\hline $\mathrm{N} 7-\mathrm{N} 6-\mathrm{C} 8-\mathrm{N} 5$ & $0.2(4)$ \\
\hline $\mathrm{C} 9-\mathrm{N} 5-\mathrm{C} 8-\mathrm{N} 6$ & $-0.4(4)$ \\
\hline $\mathrm{C} 7-\mathrm{N} 5-\mathrm{C} 8-\mathrm{N} 6$ & $-179.7(3)$ \\
\hline $\mathrm{N} 6-\mathrm{N} 7-\mathrm{C} 9-\mathrm{N} 5$ & $-0.2(4)$ \\
\hline $\mathrm{C} 8-\mathrm{N} 5-\mathrm{C} 9-\mathrm{N} 7$ & $0.4(4)$ \\
\hline $\mathrm{C} 7-\mathrm{N} 5-\mathrm{C} 9-\mathrm{N} 7$ & $179.7(3)$ \\
\hline $\mathrm{N} 9-\mathrm{N} 8-\mathrm{C} 10-\mathrm{N} 10$ & $0.5(4)$ \\
\hline $\mathrm{C} 11-\mathrm{N} 10-\mathrm{C} 10-\mathrm{N} 8$ & $-0.5(3)$ \\
\hline $\mathrm{C} 12-\mathrm{N} 10-\mathrm{C} 10-\mathrm{N} 8$ & $-179.7(3)$ \\
\hline $\mathrm{N} 8-\mathrm{N} 9-\mathrm{C} 11-\mathrm{N} 10$ & $0.0(3)$ \\
\hline $\mathrm{C} 10-\mathrm{N} 10-\mathrm{C} 11-\mathrm{N} 9$ & $0.3(3)$ \\
\hline $\mathrm{C} 12-\mathrm{N} 10-\mathrm{C} 11-\mathrm{N} 9$ & $179.5(3)$ \\
\hline $\mathrm{C} 16-\mathrm{N} 11-\mathrm{C} 12-\mathrm{C} 13$ & $-0.3(4)$ \\
\hline $\mathrm{C} 16-\mathrm{N} 11-\mathrm{C} 12-\mathrm{N} 10$ & $179.6(2)$ \\
\hline $\mathrm{C} 10-\mathrm{N} 10-\mathrm{C} 12-\mathrm{N} 11$ & 173.0 \\
\hline $\mathrm{C} 11-\mathrm{N} 10-\mathrm{C} 12-\mathrm{N} 11$ & $-6.0(4)$ \\
\hline $\mathrm{C} 10-\mathrm{N} 10-\mathrm{C} 12-\mathrm{C} 13$ & $-7.1(5)$ \\
\hline $\mathrm{C} 11-\mathrm{N} 10-\mathrm{C} 12-\mathrm{C} 13$ & $173.8(3)$ \\
\hline
\end{tabular}

$\begin{array}{ll}\mathrm{C} 18-\mathrm{N} 12-\mathrm{C} 16-\mathrm{C} 15 & 7.1(5) \\ \mathrm{C} 17-\mathrm{N} 12-\mathrm{C} 16-\mathrm{C} 15 & -173.7(3) \\ \mathrm{N} 14-\mathrm{N} 13-\mathrm{C} 17-\mathrm{N} 12 & 0.0(3) \\ \mathrm{C} 18-\mathrm{N} 12-\mathrm{C} 17-\mathrm{N} 13 & 0.0(3) \\ \mathrm{C} 16-\mathrm{N} 12-\mathrm{C} 17-\mathrm{N} 13 & -179.4(3) \\ \mathrm{N} 13-\mathrm{N} 14-\mathrm{C} 18-\mathrm{N} 12 & 0.0(3) \\ \mathrm{C} 17-\mathrm{N} 12-\mathrm{C} 18-\mathrm{N} 14 & 0.0(3) \\ \mathrm{C} 16-\mathrm{N} 12-\mathrm{C} 18-\mathrm{N} 14 & 179.4(3) \\ \mathrm{N} 16-\mathrm{N} 15-\mathrm{C} 19-\mathrm{N} 17 & -0.3(3) \\ \mathrm{C} 20-\mathrm{N} 17-\mathrm{C} 19-\mathrm{N} 15 & 0.3(3) \\ \mathrm{C} 21-\mathrm{N} 17-\mathrm{C} 19-\mathrm{N} 15 & -179.8(3) \\ \mathrm{N} 15-\mathrm{N} 16-\mathrm{C} 20-\mathrm{N} 17 & 0.0(3) \\ \mathrm{C} 19-\mathrm{N} 17-\mathrm{C} 20-\mathrm{N} 16 & -0.2(3) \\ \mathrm{C} 21-\mathrm{N} 17-\mathrm{C} 20-\mathrm{N} 16 & 180.0(3) \\ \mathrm{C} 25-\mathrm{N} 18-\mathrm{C} 21-\mathrm{C} 22 & -0.2(4) \\ \mathrm{C} 25-\mathrm{N} 18-\mathrm{C} 21-\mathrm{N} 17 & 179.6(2) \\ \mathrm{C} 19-\mathrm{N} 17-\mathrm{C} 21-\mathrm{N} 18 & 173.2(3) \\ \mathrm{C} 20-\mathrm{N} 17-\mathrm{C} 21-\mathrm{N} 18 & -7.0(4) \\ \mathrm{C} 19-\mathrm{N} 17-\mathrm{C} 21-\mathrm{C} 22 & -7.0(5) \\ \mathrm{C} 20-\mathrm{N} 17-\mathrm{C} 21-\mathrm{C} 22 & 172.9(3) \\ \mathrm{N} 18-\mathrm{C} 21-\mathrm{C} 22-\mathrm{C} 23 & 0.2(5) \\ \mathrm{N} 17-\mathrm{C} 21-\mathrm{C} 22-\mathrm{C} 23 & -179.6(3) \\ \mathrm{C} 21-\mathrm{C} 22-\mathrm{C} 23-\mathrm{C} 24 & -0.1(5) \\ \mathrm{C} 22-\mathrm{C} 23-\mathrm{C} 24-\mathrm{C} 25 & 0.0(5) \\ \mathrm{C} 21-\mathrm{N} 18-\mathrm{C} 25-\mathrm{C} 24 & 0.1(4) \\ \mathrm{C} 21-\mathrm{N} 18-\mathrm{C} 25-\mathrm{N} 19 & 179.3(2) \\ \mathrm{C} 23-\mathrm{C} 24-\mathrm{C} 25-\mathrm{N} 18 & -0.1(5) \\ \mathrm{C} 23-\mathrm{C} 24-\mathrm{C} 25-\mathrm{N} 19 & -179.1(3) \\ \mathrm{C} 27-\mathrm{N} 19-\mathrm{C} 25-\mathrm{N} 18 & -175.5(3) \\ \mathrm{C} 26-\mathrm{N} 19-\mathrm{C} 25-\mathrm{N} 18 & 1.5(4) \\ \mathrm{C} 27-\mathrm{N} 19-\mathrm{C} 25-\mathrm{C} 24 & 3.7(5) \\ \mathrm{C} 26-\mathrm{N} 19-\mathrm{C} 25-\mathrm{C} 24 & -179.3(3) \\ \mathrm{N} 21-\mathrm{N} 20-\mathrm{C} 26-\mathrm{N} 19 & -0.5(4) \\ \mathrm{C} 27-\mathrm{N} 19-\mathrm{C} 26-\mathrm{N} 20 & 0.4(4) \\ \mathrm{C} 25-\mathrm{N} 19-\mathrm{C} 26-\mathrm{N} 20 & -177.2(3) \\ \mathrm{N} 20-\mathrm{N} 21-\mathrm{C} 27-\mathrm{N} 19 & -0.1(4) \\ \mathrm{C} 26-\mathrm{N} 19-\mathrm{C} 27-\mathrm{N} 21 & -0.1(4) \\ \mathrm{C} 25-\mathrm{N} 19-\mathrm{C} 27-\mathrm{N} 21 & 177.4(3)\end{array}$

Hydrogen-bond geometry $\left(\AA,{ }^{\circ}\right)$

\begin{tabular}{lllll}
\hline$D-\mathrm{H} \cdots A$ & $D-\mathrm{H}$ & $\mathrm{H} \cdots A$ & $D \cdots A$ & $D-\mathrm{H} \cdots A$ \\
\hline $\mathrm{O} 6-\mathrm{H} 6 A \cdots \mathrm{N} 21^{\mathrm{i}}$ & $0.86(1)$ & $1.95(1)$ & $2.801(3)$ & $172(3)$ \\
$\mathrm{O} 6-\mathrm{H} 6 B \cdots \mathrm{N} 1^{\mathrm{ii}}$ & $0.86(1)$ & $2.04(1)$ & $2.878(3)$ & $166(3)$ \\
$\mathrm{O} 5-\mathrm{H} 5 A \cdots \mathrm{O} 6$ & $0.87(1)$ & $1.86(2)$ & $2.707(3)$ & $166(4)$ \\
$\mathrm{O} 5-\mathrm{H} 5 B \cdots \mathrm{O} 1^{\mathrm{iii}}$ & $0.86(1)$ & $2.01(1)$ & $2.864(4)$ & $171(4)$ \\
$\mathrm{O} 4-\mathrm{H} 4 A \cdots \mathrm{N} 14^{\mathrm{iv}}$ & $0.86(1)$ & $1.95(1)$ & $2.808(3)$ & $173(4)$ \\
$\mathrm{O} 4-\mathrm{H} 4 B \cdots \mathrm{N} 8^{\mathrm{v}}$ & $0.86(1)$ & $2.09(2)$ & $2.907(3)$ & $160(3)$
\end{tabular}


supporting information

$\begin{array}{lllll}\mathrm{O} 3-\mathrm{H} 3 B \cdots \mathrm{O} 5 & 0.86(1) & 2.03(2) & 2.868(4) & 166(4) \\ \mathrm{O} 3-\mathrm{H} 3 A \cdots \mathrm{O} 4 & 0.85(1) & 2.01(1) & 2.855(3) & 173(3) \\ \mathrm{O} 2-\mathrm{H} 2 B \cdots \mathrm{O} 4 & 0.86(1) & 2.00(1) & 2.851(4) & 172(4) \\ \mathrm{O} 2-\mathrm{H} 2 A \cdots \mathrm{O} 1 & 0.87(1) & 2.03(1) & 2.886(3) & 171(3) \\ \mathrm{O} 1-\mathrm{H} 1 B \cdots \mathrm{N} 15^{\text {vi }} & 0.86(1) & 2.05(2) & 2.861(3) & 158(3) \\ \mathrm{O} 1-\mathrm{H} 1 A \cdots \mathrm{N} 7 & 0.86(1) & 1.97(1) & 2.820(3) & 171(3)\end{array}$

Symmetry codes: (i) $x-1, y, z$; (ii) $-x, y+1 / 2,-z+3 / 2$; (iii) $x,-y+3 / 2, z-1 / 2$; (iv) $-x+2, y-1 / 2,-z+3 / 2$; (v) $x+1, y, z$; (vi) $-x+1, y+1 / 2,-z+3 / 2$. 